\title{
Nanopatterning in a compact setup using table top extreme ultraviolet lasers
}

\author{
P.W. WACHULAK ${ }^{* 1}$, M.G. CAPELUTO ${ }^{2}$, C.S. MENONI ${ }^{1}$, J.J. ROCCA ${ }^{1}$, and M.C. MARCONI ${ }^{1}$ \\ ${ }^{1}$ NSF ERC for Extreme Ultraviolet Science \& Technology and Department of Electrical \\ and Computer Engineering, Colorado State University, USA \\ ${ }^{2}$ Departamento de Fisica, Facultad de Ciencias Exactas y Naturales Universidad de Buenos Aires,
} Argentina

\begin{abstract}
The recent development of table top extreme ultraviolet (EUV) lasers have enabled new applications that so far were restricted to the use of large facilities. These compact sources bring now to the laboratory environment the capabilities that will allow a broader application of techniques related to nanotechnology and nanofabrication. In this paper we review the advances in the utilization of EUV lasers in nanopatterning. In particular we show results of the nanopatterning using a table-top capillary discharge laser producing 0.12-mJ laser pulses with 1.2-ns time duration at a wavelength $\lambda=46.9 \mathrm{~nm}$. The nanopatterning was realized by interferometric lithography using a Lloyd's mirror interferometer. Two standard photoresists were used in this work, polymethyl methacrylate (PMMA) and hydrogen silsesquioxane (HSQ). Pillars with a full width half maximum (FWHM) diameter of $60 \mathrm{~nm}$ and holes with FWHM diameter of $130 \mathrm{~nm}$ were obtained over areas in excess of $500 \times 500 \mathrm{\mu m}^{2}$.
\end{abstract}

Keywords: nanopatterning, interferometric lithography, EUV lasers, photoresist.

\section{Introduction}

Nanotechnology is a broad field covering different interdisciplinary research in physics, chemistry, biology, etc. This exciting discipline is now driving a dramatic expansion in science and technology. This is a new field that deals with phenomena that became evident when the dimensions of the systems under consideration approaches the typical distances of fundamental physical or chemical interactions. Novel applications with important technological derivations are now possible exploiting the unique behaviour of the "nanoworld". The compelling need to understand and harness the phenomena occurring at the nanometer scale has also driven a necessity for new techniques and tools to efficiently fabricate nano-structures in a controlled manner.

There are basically two different techniques employed in the fabrication of nanostructures. The two basic approaches are usually designed as the "top down" where the surface is modified by controlling beams of photons or particles, or the "bottom up" approach, where the nanostructure is constructed from the surface of the substrate taking advantage of elemental physical and chemical properties of the surface that generates self assembled nanostructures.

The top down approach is probably the most widely applied and is the one used by the semiconductor industry for production of integrated circuits. Typically, it consists of a

\footnotetext{
*e-mail: wachulak@engr.colostate.edu
}

complicated sequence of operations including photolithography, etching, coating, growth, etc, that gives rise to the complex 3D structures that now are fabricated by the multibillion dollar electronic industry. In the bottom up approach, the strategy relies on using and controlling chemical and physical characteristics of the surface to generate the desired nano-structure. The physics and chemistry reactions that take place at the surface are responsible for the nanopatterning. An example of this approach is self assembly of nanospheres, polymers or the spontaneous organized growth of different chemical species in a surface.

A driving force in nanotechnology is undoubtedly the micro and nano-electronic industry. In order to follow Moore's law, semiconductor industry had invested enormous amount of resources to produce nowadays integrated circuits with a critical size below $50 \mathrm{~nm}$. However, tools utilized in production of integrated circuits are far beyond the possibility to be utilized in low scale research and development oriented nanofabrication facilities. Research oriented developments had rely in alternative techniques in which one of the important characteristics is the simplicity and the versatility necessary for prototyping or for proof of principle experiments.

Electron beam lithography and photolithography are probably the two approaches that provide the most versatility in small scale nano-fabrication. Both rely on the activation of a suitable sensitive material, usually a polymeric photoresist by electrons or photons. The activated polymer 
in which the nanopattern is defined then serves as a printing mask to transfer the nanostructure onto the substrate surface by different techniques. Electron beam lithography had proved to be a powerful tool to fabricate arbitrary shape nanostructures with critical size down to few tens of nanometers. However, it is time consuming due to the inherent serial process of the writing. On the other hand, photons can be manipulated to form images and activate the photoresist as the first step in the fabrication sequence. The projection photolithography approach is an intrinsically parallel process, however, the limiting factor is the sophisticated and expensive optical components necessary to achieve images with sub-100 $\mathrm{nm}$ resolution. In addition to this inconvenience, there is a fundamental limit imposed by diffraction that restricts the resolution to the wavelength scale.

Reducing the wavelength is a direct path to reduce the size of the features that can be printed. This is the reason why photolithography is typically implemented with UV lasers and UV incoherent sources. To further reduce the size of the printed feature, improvements can be implemented like multiple exposures or immersion photolithography but ultimately, the wavelength of the illumination presents an insurmountable barrier to the minimum size of the feature that can be printed.

Harnessing light to print wavelength-size features has been instrumental in the realization of electronic devices. The adoption of EUV lithography as the tool to fabricate the next generation of integrated circuits is yet another example of the importance that light will have in the implementation of the new patterning tools. On this path, compact EUV laser sources open new exciting possibilities to realize efficient nano-patterning in a compact (table top size) setup with comparable capabilities to systems now accessible only with synchrotron sources. The development of compact efficient EUV sources with a wavelength 10 to 50 times smaller than visible lasers facilitated the demonstration of a large number of applications. Those include among others lithography, interferometry, microscopy, holography and nano-ablation.

In this paper we will review the last achievements in the development of a compact table-top nanopatterning tool based on the combination of the well established interferometric lithography technique and a table top extreme ultraviolet laser.

\section{Interferometric lithography (IL)}

Interferometric lithography is a well established methodology to fabricate periodic nano-structures over large areas. The characteristic advantages of this approach as compared to other lithographic methods relies on the absence of expensive and sophisticated optical systems and in the intrinsic parallelism of the process that produces a larger throughput as compared with traditional electron beam writing.
The basic concept is to combine two or more coherent light beams and made them to interfere in the surface of a photoresist coated substrate. In the overlapping region, an interference pattern is created with intensity distribution characterized by the wavelength used for the illumination, the number of combined laser beams, and other parameters like coherence of the source, polarization, etc. that activates the photoresist with a pre-designed periodic pattern. The simplest case of two beams is schematically depicted in Fig. 1. In this case, the pattern produced is a sinusoidal grating with the period

$$
d=\lambda /(2 \sin (\theta)) .
$$

For example, using light beams with $257-n m$ wavelength and the incident angle $\theta$ of $80^{\circ}$, lines and spaces of a period of $130 \mathrm{~nm}$ can be obtained, roughly half what it is attainable with a state-of-the-art 248-nm projection tool.

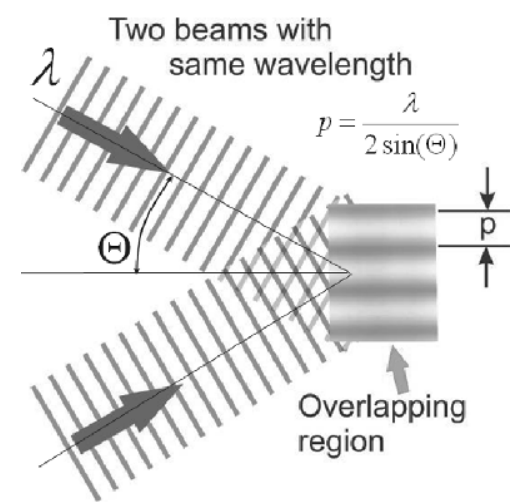

Fig. 1. Schematic description of the interference lithography technique used in nanopatterning.

Equation (1) indicates that the ultimate resolution in IL is given by the wavelength of the illumination. A direct path to reduce the size of the feature that can be printed with IL is to use shorter wavelengths. This was the main motivation for using argon and fluorine lasers in the ultraviolet (UV) and deep ultraviolet (DUV) regions of the electromagnetic spectrum, as well as synchrotron radiation that can be tuned from hard X-rays to EUV region of spectrum.

The comparatively simplicity of interference lithography makes this approach a very convenient way to fabricate nanostructures in a simple manner. Different groups followed diverse approaches to achieve smaller feature size in the printed samples. One approach is using short wavelength lasers and filtered synchrotron radiation as the illuminating source to reduce the feature size that can be attained. Also multiple diffractive masks, amplitude and wavefront division interferometers configurations were used to generate more complex patterns, different than simple lines. Another approach is to use special immersion optics with the aim to increase the numerical aperture (NA) of the projecting optics and improve the resolution. The ultimate limit for the smallest feature that can be print in the photoresist is determined by the relationship 


$$
d=\frac{k \lambda}{N A},
$$

where the constant $k$ accounts for the characteristics of the illumination and its value may change from $\sim 0.34$ up to 1 , depending on the degree of coherence of the illumination, the illumination spectrum, and the test used for the resolution assessment, and $\lambda$ is the wavelength of the illumination [1]. Consequently the main strategies to reduce the size of the printed feature are to reduce the illumination wavelength or to increase the $N A$ of the printing optical system.

The illumination wavelength can be reduced using $\mathrm{Ar}$, F or ArF lasers. Savas et al. using an $\operatorname{ArF}$ laser $(\lambda=193$ $\mathrm{nm}$ ) and phase gratings demonstrated sub- $100 \mathrm{~nm}$ periodic structures patterned in the surface of the photoresist $[2,3]$. The highly coherent beam from the ArF laser allowed for patterning areas as big as $10 \mathrm{~cm}^{2}$. Zaidi et al. using multiple exposure interferometric lithography showed the possibility of patterning one and two dimensional patterns with $0.6-\mu \mathrm{m}$ period [4]. Hinsberg et al. described the design and operating characteristics of a deep ultraviolet (DUV) interferometric lithography tool using for illumination a continuous-wave beam at $257 \mathrm{~nm}$ wavelength [5].

Further, feature size reduction can be achieved utilizing even lower wavelength radiation from synchrotron facilities. Synchrotrons provide a large photon flux and tunable output, but the beam spatial and temporal coherence are much lower than those typically obtained with laser sources. Additional filtering is often necessary to obtain good interference contrast and large patterned areas. This necessary filtering has as immediate consequence in a serious flux reduction. Regardless this inconvenience, synchrotrons were always an attractive short wavelength source for leading-edge IL experiments. Additionally EUV radiation has its own challenges that arise from the high absorption coefficients that makes difficult to achieve optical elements with high throughputs in this region of the spectrum. That is the reason why most of the patterning tools based on IL are rather simple and use the minimum possible number of reflecting surfaces. One very convenient configuration is the simple Lloyd's mirror interferometer. Solak et al. have developed a EUV IL system based on an undulator radiation and a Lloyd's mirror. A record 19-nm line and space patterns (38 nm period) were achieved using this system with 13.4-nm wavelength in polymethyl methacrylate photoresist [6,7].

Extensions of the two beams interference lithography to multiple beams interference was reported by Fernandez et al. [8,9]. A multiple-grating mask was used to generate several coherent beams that allowed the fabrication of arrays of holes or pillars in rectangular or circular patterns as small as $100 \mathrm{~nm}$. Multiple beam EUV interference lithography systems were developed to fabricate two-dimensional periodic structures in square matrices or in circular patterns using specially designed diffraction masks. These specially fabricated masks allowed for great versatility in the shape, period, and configuration of the two dimensional printing [10-13].
Another way to increase the resolution of the system and be able to pattern smaller features is increasing the numerical aperture of the optical system. The typical example can be the projection tools with high numerical aperture objectives and large acceptance angle. An alternative approach to increase the NA is by using immersion lithography. The use of immersion media liquids to extend optical resolution is well known and was used for a long time in oil-immersion optical microscopy. Introducing the liquid with high index of refraction between the sample and the objective allows to increase the numerical aperture and to improve the resolution in terms of Rayleigh criterion. Frauenglass et al. using 22x reduction imaging interferometric lithography test-bed with $N A=0.9$ lens demonstrated printing of an arbitrary 86-nm half-pitch patterns with illumination from a frequency doubled Ar-ion laser $(\lambda=244 \mathrm{~nm})$ [14]. Through using de-ionized water smaller minimum feature sizes can be achieved in compare with ones achieved without immersion. Raub et al. showed that through the use of exposure media (purified water with $n$ of $1.44)$ at $193-\mathrm{nm}$ wavelength it is possible to reduce the minimum feature sizes compared with traditional air/vacuum exposures media by a factor of $44 \%$. They also demonstrated 45-nm half-period lines patterned into resist using liquid immersion lithography (LIL) with a $N A=1.18$ and $\lambda$ $=213 \mathrm{~nm}$ [15].

Interference lithography is a very simple approach for EUV photolithography that allows patterning of relatively large areas with short exposure times. This method is useful in applications where periodically repeated cell structures, holes, pillars, lines or other periodic features are needed for example in optical filters, photonic crystals, display units, electronic memories and logic circuits as well as magnetic storage devices.

\section{Table top extreme ultraviolet (EUV) capillary discharge laser}

The versatility of IL to print and fabricate nanometer size periodic structures was particularly benefited with the demonstration of table top EUV lasers that enabled in compact laboratory-size setups capabilities so far exclusively reserved to large synchrotron facilities. In this paper we will describe a series of experiments where the illumination source used is a compact $\lambda=46.9 \mathrm{~nm}$ table top discharge-pumped capillary Ne-like Ar laser [16,17]. The laser is a very compact unit occupying only a $10.5 \mathrm{~m}^{2}$ footprint on an optical table $[18,19]$. Lasing was obtained in the $46.9 \mathrm{~nm} 3 \mathrm{~s}^{1} \mathrm{P}_{1}-3 \mathrm{p}^{1} \mathrm{~S}_{0}$ transition of neon-like Ar after exciting Ar filled alumina capillary $3.2 \mathrm{~mm}$ inner diameter with a current pulse having an amplitude of $\approx 24 \mathrm{kA}$, a $10 \%$ to $90 \%$ rise time of $\approx 25 \mathrm{~ns}$ and a first half-cycle duration of $\approx 110 \mathrm{~ns}$. The fast current pulse was produced by discharging a water dielectric cylindrical capacitor through a spark gap switch connected in series with the capillary load. The current pulse rapidly compresses the plasma column to 
achieve a dense and hot filamentary plasma channel where a population inversion is created by strong monopole electron impact excitation of the laser upper level and rapid radiative relaxation of the laser lower level. The water serves as a liquid dielectric for the capacitor and also cools the capillary. A continuous flow of $\mathrm{Ar}$ is injected in the front of the capillary and an optimum Ar gas pressure of 490 mTorr is maintained in the capillary channel. Laser was configured to produce pulses having the energy of about $0.2 \mathrm{~mJ}$ and $1.2 \mathrm{~ns}$ FWHM duration. When operated at repetition rates of several $\mathrm{Hz}$, this table-top laser can produce EUV beams with average powers exceeding $1 \mathrm{~mW}$ and a high degree of spatial and temporal coherence. The 27-cm long plasma column generates a laser beam that has a radius of coherence of approximately $570 \mu \mathrm{m}$ measured in the experiment chamber located at $1.7 \mathrm{~m}$ from the end of the capillary [20,21]. The spatial coherence length can be further increased using longer capillaries also increasing the energy per pulse delivered. The laser beam temporal coherence length is greater than $470 \mu \mathrm{m}$ as determined by its line width $\Delta \lambda / \lambda<1 \times 10^{-4}$.

\section{Nanopatterning using compact patterning tool based on Lloyd's mirror interferometer}

The interferometric lithography nanopatterning tool was implemented by illuminating a flat mirror in the Lloyd's configuration with the EUV laser output. In this configuration, part of the laser beam reflects from the mirror at the incidence angle $\theta$ and interferes with the remaining un-deflected part of the beam, as illustrated in Fig. 2. Beam interference gives rise to a sinusoidal intensity pattern of the period $d$, defined by the wavelength of the light $\lambda$ and the incidence angle $\theta$ according to $d=\lambda /(2 \sin \theta)$.

Figure 2 shows the set up utilized in this experiment. A rectangular $30 \times 50 \mathrm{~mm}^{2} \mathrm{Cr}$ coated flat mirror was mounted at grazing incidence in front of the laser beam on a pivoting platform, with its axis coincident with the farther edge of the mirror. The sample, consisting of a $\mathrm{Si}$ wafer coated with polymethyl methacrylate, was mounted at this edge in a motorized rotation stage. Controlling the rotation angle $\alpha$ around an axis normal to the sample's surface allows for multiple exposures in a two steps procedure as schematically depicted in Fig. 3. A translation stage was used to retract the sample from the mirror's edge before each rota-
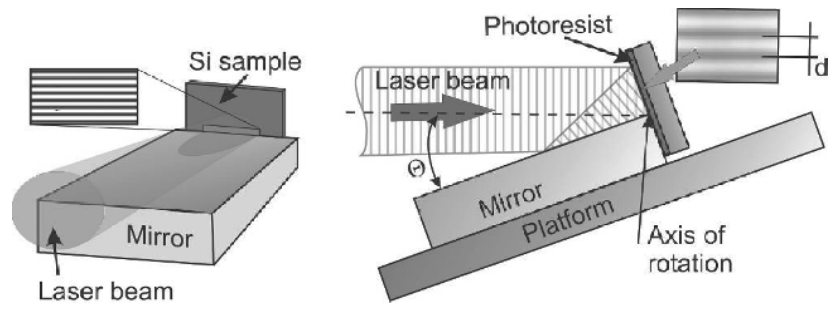

Fig. 2. Schematic description of a Lloyd's mirror setup. Single step exposure results generating the interference pattern with lines parallel to the edge of the mirror and with the period $d$.

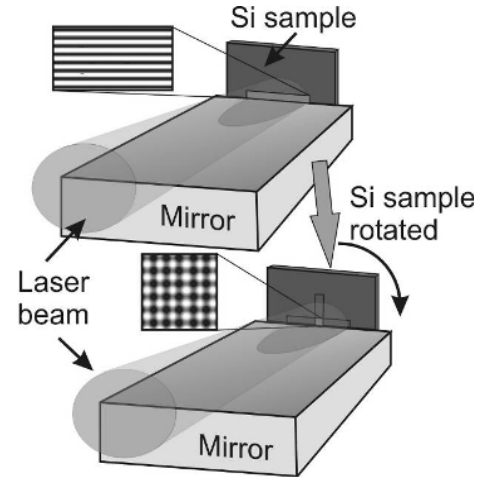

Fig. 3. Two steps exposure. The sample is rotated an arbitrary angle around the axis perpendicular to the sample surface which allows to obtain different motifs.

tion. This procedure was carried out without necessity to break vacuum thus, assuring similar conditions for both exposures and increasing significantly the throughput of the patterning process. The entire system was mounted on a motorized pivoting platform that allows changing the incidence angle $\theta$ and in this way the period of the patterned features. The Lloyd mirror interferometer system was housed in a vacuum chamber $0.45 \times 0.55 \times 0.40 \mathrm{~m}^{3}$ that was differentially pumped in respect to the laser to maintain a pressure of approximately $10^{-5}$ Torr. The entire EUV interferometry instrument is very compact, the footprint is about $0.7 \times 2.6 \mathrm{~m}^{2}$.

\subsection{Patterning using PMMA photoresist}

An ultra thin layer of polymethyl methacrylate, approximately 30-nm thick, was spun on the surface of a Si wafer. This was accomplished by spin coating, the substrate with diluted 1\% PMMA in anisole at 5000 RPM in a standard spin coater. Samples were pre-baked for 1 hour at $60^{\circ} \mathrm{C}$ to remove the solvent using standard hotplate with $+/-0.5^{\circ} \mathrm{C}$ temperature control. After the exposure, the PMMA was developed using the standard procedure. The samples were immersed in a 1:3 solution of MIBK-methyl isobutyl ketone (4-Methyl-2-Pentanone) with IPA-isopropyl alcohol for 35 seconds and rinsed with IPA. Finally, the sample was dried using compressed nitrogen.

The photon dose applied in each exposure, corresponding to the energy per unit area, allows a degree of control over the shape of the imprinted features. The thickness of the photoresist that remains in the exposed areas after the developing process (residual thickness) is a linear function of the applied dose. For PMMA, the residual thickness was measured by Junarsa et al. [22]. Using these data we calculated how the patterned profile obtained in a double exposure would change as a function of the applied dose. Figure 4(a) shows the calculated profile obtained in a PMMA layer for a low dose exposure of $110 \mathrm{~mJ} \mathrm{~cm}^{-2}$. The pattern was calculated by the incoherent addition of two identical interference patterns rotated by an angle $\alpha=\pi / 2$. Such a dose of $110 \mathrm{~mJ} \mathrm{~cm}-2$ at the sample plane can be obtained 


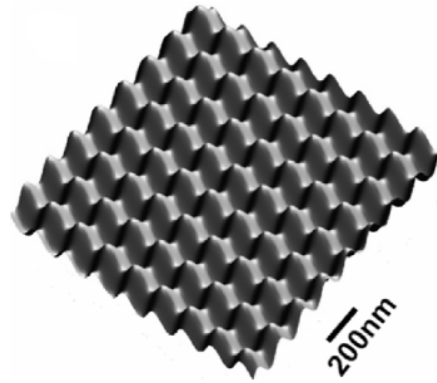

(a)

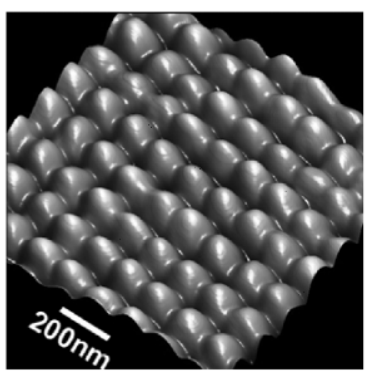

(b)

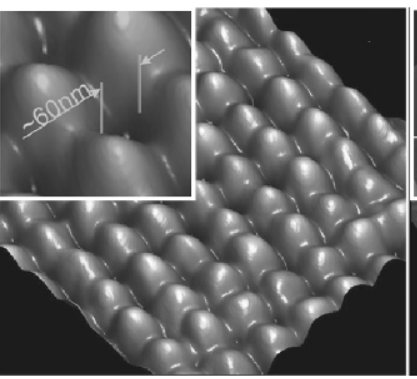

(a)

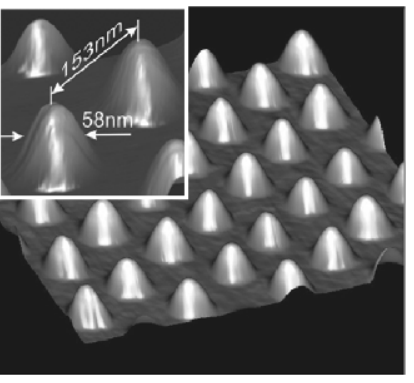

(b)

Fig. 4. Simulated (a) and fabricated (b) arrays of holes patterned in PMMA by double exposure with a Lloyd's mirror configuration, $\alpha=\pi / 2$ and low $\left(110 \mathrm{~mJ} \mathrm{~cm}^{-2}\right)$ photon dose.

with about 40 laser shots. The simulation shows that the photoresist is only activated in the regions of overlapping of two interference maxima (region with the highest dose), developing in these regions small holes. A similar pattern was obtained experimentally, shown in Fig. 4(b).

If the applied dose is increased to $166 \mathrm{~mJ} \mathrm{~cm}^{-2}$ (high dose, about 60 laser shots), the loci in the sample surface with sufficient exposure to activate the photoresist are broader. In this case, the activated volume generates after developing of the photoresist a regular array of trenches in two perpendicular directions leaving small non activated spots at the intersections where the applied dose was smaller. Figure 5(a) shows the calculated pattern corresponding to the high dose exposure. The experimental pattern fabricated in the PMMA surface is shown in Fig. 5(b) and corresponds to a very uniform array of cone-shaped pillars that are similar to the simulation. The period of the patterned features can be continuously changed by changing the incidence angle $\theta$ at which the laser beam illuminated the edge of the mirror. Figure 6(a) shows the details of the measured hole pattern with hole diameter $\sim 60 \mathrm{~nm}$ and $160 \mathrm{~nm}$ period, while Fig. 6(b) shows the details of the measured profile of the small pillars with FWHM $\sim 58 \mathrm{~nm}$ and the period $153 \mathrm{~nm}$. The height of the cones and the depth of the holes pattern correspond to the penetration depth of the $46.9 \mathrm{~nm}$ photons in the PMMA photoresist, approximately $30 \mathrm{~nm}$ [23].

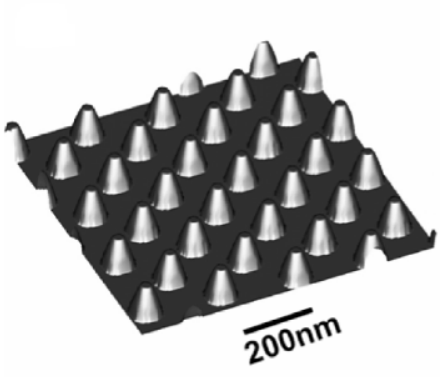

(a)

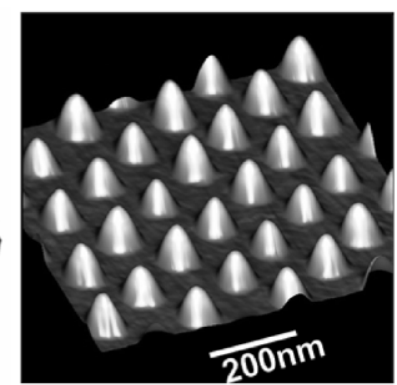

(b)

Fig. 5. Simulated (a) and fabricated (b) arrays of cone-shaped nano-dots patterned in PMMA by double exposure with a Lloyd's mirror configuration, with $\alpha=\pi / 2$ and high $\left(166 \mathrm{~mJ} \mathrm{~cm}^{-2}\right)$ photon dose.

Fig. 6. Magnified view (a) of a smaller area of the array in (b), showing the hole pattern. The FWHM of the holes is around $60 \mathrm{~nm}$, and the period $160 \mathrm{~nm}$. Magnified view (b) of a smaller area of the array in (b), showing the cone-shaped nano-dots. The FWHM of the nano-dot is

approximately $60 \mathrm{~nm}$ and the period is nominally $150 \mathrm{~nm}$.

The rotation angle between the two consecutive exposures allowed also printing different shaped features. If the sample was rotated an angle $\alpha=\pi / 2$, the array developed in the surface of the photoresist had a square distribution as shown in Fig. 7(a). Instead, if the rotation angle was different, for example $\alpha=\pi / 6$, the array of elongated dots (ovals) was fabricated as shown in Fig. 7(b).

\subsection{Patterning using HSQ photoresist}

The shallow penetration depth in PMMA imposes a limitation for the further processing of the underlying substrate. A promising solution to this problem is the utilization of an alternative photoresist. Hydrogen silsesquioxane has been extensively used as a negative tone photoresist for e-beam lithography, with demonstrated spatial resolution around 10 to $12 \mathrm{~nm}$ [24]. HSQ was also investigated for its applicability in photolithography. The behaviour of the resist under the illumination with different wavelengths was previously studied, showing that HSQ is non sensitive to visible and UV light down to $193 \mathrm{~nm}$ wavelength. However, at $157 \mathrm{~nm}$ lithographic activity was observed although with high pho-

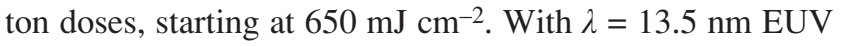
radiation, the negative tone behaviour was first reported to

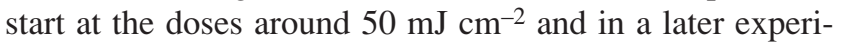
ment at $11.5 \mathrm{~mJ} \mathrm{~cm}^{-2}[22,25]$.

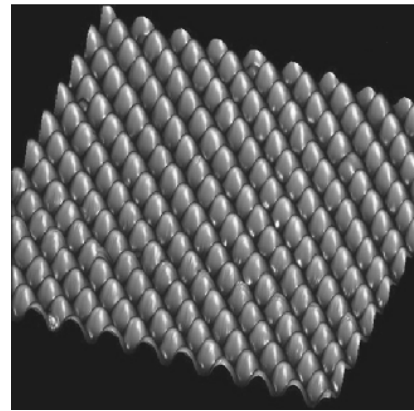

(a)

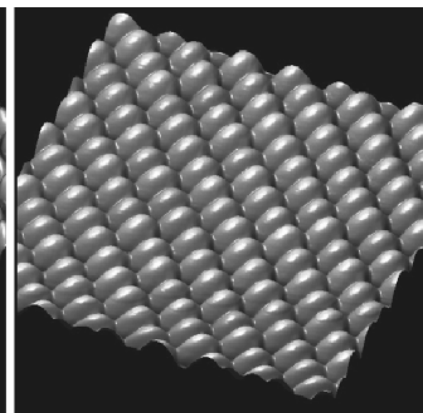

(b)
Fig. 7. The arrays of nanodots patterned in PMMA photoresist (a) with the angle between exposures equal to $\alpha=\pi / 2$ and (b) $\alpha=\pi / 6$. 

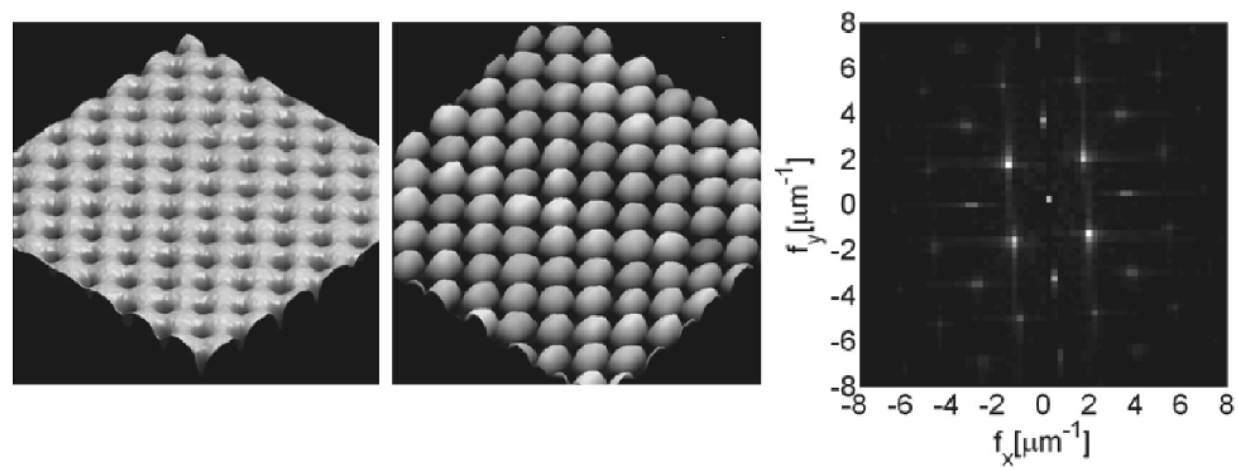

Fig. 8. Array of holes $130 \mathrm{~nm}$ FWHM and $100 \mathrm{~nm}$ deep fabricated in HSQ with a high illumination dose (a), array of nanodots obtained with low illumination dose (b), two dimensional Fourier transform of the image (a) showing only significant contributions at the hole spacing spatial frequency and its harmonics (c).

HSQ chemical composition (silicon, hydrogen and oxygen) makes HSQ more transparent to $\lambda=46.9 \mathrm{~nm}$ photons as compared with the carbon-based photoresist PMMA. That results in a measured penetration depth exceeding 150 $\mathrm{nm}$, allowing in this way printing of larger aspect ratio features. HSQ has also a lower activation threshold (about 3 times smaller than PMMA) which significantly reduced the exposure time necessary to print different patterns.

HSQ was spin coated on the surface of a Si wafer to obtain the photoresist thickness around $150 \mathrm{~nm}$, then prebaked at $115^{\circ} \mathrm{C}$ for 1 minute to evaporate the solvent. The coated samples were exposed to different doses, developed with 2.38\% TMAH (tetramethylammonium hydroxide) for $30 \mathrm{~s}$ and rinsed with DI water. Multiple exposure IL was implemented with the HSQ coated samples utilizing the same experimental set up based on the Lloyd's mirror.

Changing the photon flux applied in each exposure gives also flexibility in printing different features. For high photon flux (approximately $14 \mathrm{~mJ} \mathrm{~cm}^{-2}$ ), corresponding to the dose at the resist surface approximately $50 \mathrm{~mJ} \mathrm{~cm}^{-2}$, the resist was activated in wide strips. Between them after developing small holes were fabricated. Figure $8(a)$ is the AFM scan showing a $4 \times 4 \mu \mathrm{m}^{2}$ section of the array of holes $130 \mathrm{~nm}$ FWHM and $100 \mathrm{~nm}$ deep patterned with this high flux. Reducing the flux to approximately $3.5 \mathrm{~mJ} \mathrm{~cm}^{-2}$, corresponding to a dose in the resist surface approximately $13.6 \mathrm{~mJ} \mathrm{~cm}^{-2}$, the photoresist is only activated in small volumes in the intersections of the fringes corresponding to the maxima of interference, developing in this case an array of small pillars, as shown in Fig. 8(b). The versatility of this IL set up allows changing the feature (holes or dots) very easily by changing the applied photon flux and the periodicity by changing the incidence angle on the mirror in the similar way as it was done previously for PMMA photoresist. The size and distribution of the holes and nano-dots in the array were very uniform as it can be observed in the 2D Fourier transform of the AFM image. This plot, shown in Fig. 8(c), has significant contributions only at the spatial frequency and its harmonics corresponding to the hole spacing.
The contrast attainable by interferometric lithography also depends on the intensities of the two interfering beams that change the visibility of the interference fringes. In the Lloyd's mirror configuration one of the beams is reflected, and consequently its intensity is modified by the reflection coefficient of the mirror's surface. This coefficient depends also on the light polarization and the incidence angle. Another factor that can reduce the intensity in the reflected beam is scattering at the mirror's surface. Detailed analysis of these factors in a former experiment indicated that the loss in contrast due to polarization and angle dependence is only important at incidence angles $\theta>7^{\circ}$ that corresponds to patterns period below $200 \mathrm{~nm}$ [26]. Also at these larger incidence angles the scattering in the reflecting surface degrades the reflectivity of the beam and the interference contrast. In the present experiment the incidence angle in the Lloyd's mirror was $\theta<7^{\circ}$ and consequently we should not expect any significant degradation in the contrast of the interference pattern due to this effect.

\section{Conclusions}

In conclusions, we have realized nanometer-scale patterning using a table-top system by interferometric lithography. Using a multiple exposure Lloyd's mirror configuration dense arrays of holes and nanodots with feature size as small as $60 \mathrm{~nm}$ were imprinted in PMMA photoresist over areas exceeding $500 \times 500 \mu \mathrm{m}^{2}$ using a $46.9 \mathrm{~nm}$ wavelength laser. The periodicity of the structures can be controlled by changing the incidence angle at the mirror while the shape of the feature can be varied by changing the applied dose. We have also realized nanometer-scale patterning in HSQ photoresist using a table-top system with a table top EUV laser and interferometric lithography. Dense arrays of holes and nanodots with a modulation depth approximately $100 \mathrm{~nm}$ were obtained. The periodicity of the structures was controlled by changing the incidence angle of laser beam at the mirror while changing the applied dose and the developing time made possible the printing of holes or dots. We found that HSQ has sensitivity at $\lambda=$ $46.9 \mathrm{~nm}$ illumination starting the photolithographic activity at doses of approximately $14 \mathrm{~mJ} \mathrm{~cm}^{-2}$ at the surface of the photoresist. We also measured a penetration depth of approxi- 
mately $150 \mathrm{~nm}$, almost one order of magnitude larger than the penetration depth obtained at the same wavelength in PMMA.

These results show that table-top EUV lasers combined with interferometric lithography schemes are very useful compact alternative for patterning nanometer size features. The table top EUV-IL approach takes advantages of compact table top EUV lasers and brings into the laboratory environment all of the advantages of this technique which implementation was previously restricted to large synchrotron facilities.

\section{Acknowledgements}

This work was supported under the NER program, NSF Award DMI-0508484 utilizing facilities from the NSF ERC for Extreme Ultraviolet Science and Technology, Award Number EEC-0310717. MGC acknowledges the support through a fellowship from CONICET.

\section{References}

1. J. Heck, D.T. Attwood, W. Meyer-Ilse, and E.H. Anderson, "Resolution determination in X-ray microscopy: an analysis of the effects of partial coherence and illumination spectrum", J. X-Ray Sci. Technol. 8, 95-104 (1998).

2. T.A. Savas, M.L. Schattenburg, J.M. Carter, and H.I. Smith, "Large-area achromatic interferometric lithography for 100-nm period gratings and grids", J. Vac. Sci. Technol. B14, 4167-4170 (1996).

3. T.A. Savas, S.N. Shah, M.L. Schattenburg, J.M. Carter, and H.I. Smith, "Achromatic interferometric lithography for 100-nm-period gratings and grids", J. Vac. Sci. Technol. B13, 2732-2735 (1995).

4. S.H. Zaidi and S.R.J. Brueck, "Multiple exposure interferometric lithography", J. Vac. Sci. Technol. B11, 658-666 (1993).

5. W. Hinsberg, F.A. Houle, J. Hoffnagle, M. Sanchez, G. Wallraff, M. Morrison, and S. Frank, "Deep-ultraviolet interferometric lithography as a tool for assessment of chemically amplified photoresist performance", J. Vac. Sci. Technol. B16, 3689-3694 (1998).

6. H.H. Solak, D. He, W. Li, and F. Cerrina, "Nanolithography using extreme ultraviolet lithography interferometry: $19 \mathrm{~nm}$ lines and spaces", J. Vac. Sci. Technol. B17, 3052-3057 (1999).

7. H.H. Solak, D. He, W. Li, S. Singh-Gasson, F. Cerrina, B.H. Sohn, X.M. Yang, and P. Nealey, "Exposure of $38 \mathrm{~nm}$ period grating patterns with extreme ultraviolet interferometric lithography", Appl. Phys. Lett. 75, 2328-2330 (1999).

8. A. Fernandez, J.Y. Decker, S.M. Herman, D.W. Phillion, D.W. Sweeney, and M.D. Perry, "Methods for fabricating arrays of holes using interference lithography", J. Vac. Sci. Technol. B15, 2439-2443 (1997).

9. A. Fernandez and D.W. Phillion, "Effects of phase shifts on four-beam interference patterns", Appl. Optics 37, 473-478 (1998).

10. H.H. Solak, "Space-invariant multiple-beam achromatic EUV interference lithography", Microelectron. Eng. 78/79, 410-416 (2005).

11. H.H. Solak, "Nanolithography with coherent extreme ultraviolet light”, J. Phys. D Appl. Phys. 39, R171-R188 (2006).
12. H.H. Solak and C. David, "Patterning of circular structure arrays with interference lithography", J. Vac. Sci. Technol. B21, 2883-2887 (2003).

13. H.H. Solak, C. David, J. Gobrecht, L. Wang, and F. Cerrina, "Multiple-beam interference lithography with electron beam written gratings", J. Vac. Sci. Technol. B20, 2844-2848 (2002).

14. A. Frauenglass, S. Smolev, A. Biswas, and S.R.J. Brueck, "244-nm imaging interferometric lithography", J. Vac. Sci. Technol. B22, 3465-3469 (2004).

15. A.K. Raub, A. Frauenglass, S.R.J. Brueck, W. Conley, R. Dammel, A. Romano, M. Sato, and W. Hinsberg, "Imaging capabilities of resist in deep ultraviolet liquid immersion interferometric lithography", J. Vac. Sci. Technol. B22, 3459-3464 (2004).

16. J.J. Rocca, V. Shlyaptsev, F.G. Tomasel, O.D. Cortazar, D. Hartshorn, and J.L.A. Chilla, "Demonstration of a discharge pumped table-top soft X-ray laser", Phys. Rev. Lett. 73, 2192-2195 (1994).

17. J.J. Rocca, F.G. Tomasel, M.C. Marconi, V.N. Shlyaptsev, J.L.A. Chilla, B.T. Szapiro, and G. Giudice, "Dischargepumped Soft-X-ray laser in neao-like argon", Phys. Plasmas 2, 2547-2554 (1995).

18. B.R. Benware, C.H. Moreno, D.J. Burd, and J.J. Rocca, "Operation and output pulse characteristics of an extremely compact capillary-discharge tabletop soft-x-ray laser", Opt. Lett. 22, 796-798 (1997).

19. C.D. Macchietto, B.R. Benware, and J.J. Rocca, "Generation of millijoule-level soft-x-ray laser pulses at a 4-Hz repetition rate in a highly saturated tabletop capillary discharge amplifier", Opt. Lett. 24, 1115-1117 (1999).

20. Y. Liu, M. Seminario, F.G. Tomasel, C. Chang, J.J. Rocca, and D.T. Attwood, "Achievement of essentially full spatial coherence in a high-average-power soft-x-ray laser", Phys. Rev. A6303, (2001).

21. Y.W. Liu, M. Seminario, F.G. Tomasel, C. Chang, J.J. Rocca, and D.T. Attwood, "Spatial coherence measurement of a high average power table-top soft X-ray laser", J. Phys. IV 11(PR2), 123-126 (2001).

22. I. Junarsa, M.P. Stoykovich, P.F. Nealey, Y.S. Ma, and F. Cerrina, "Hydrogen silsesquioxane as a high resolution negative-tone resist for extreme ultraviolet lithography", J. Vac. Sci. Technol. B23, 138-143 (2005).

23. P.W. Wachulak, M.G. Capeluto, M.C. Marconi, C.S. Menoni, and J.J. Rocca, "Patterning of nano-scale arrays by table-top extreme ultraviolet laser interferometric lithography”, Opt. Express 15, 3465-3469 (2007).

24. I.B. Baek, J.H. Yang, W.J. Cho, C.G. Ahn, K. Im, and S. Lee, "Electron beam lithography patterning of sub-10 nm line using hydrogen silsesquioxane for nanoscale device applications", J. Vac. Sci. Technol. B23, 3120-3123 (2005).

25. M. Peuker, M.H. Lim, H.I. Smith, R. Morton, A.K. van Langen-Suurling, J. Romijn, E. van der Drift, and F. van Delft, "Hydrogen silsesquioxane, a high-resolution negative tone e-beam resist, investigated for its applicability in photon-based lithographies", Microelectron. Eng. 61/2, 803-809 (2002).

26. M.G. Capeluto, G. Vaschenko, M. Grisham, M.C. Marconi, S. Luduena, L. Pietrasanta, Y.F. Lu, B. Parkinson, C.S. Menoni, and J.J. Rocca, "Nanopatterning with interferometric lithography using a compact $\lambda=46.9$-nm laser", IEEE T. Nanotechnol. 5, 3-7 (2006). 